\title{
Depressão na Gestação e no Pós-Parto e a Responsividade Materna Nesse Contexto
}

\section{Depression in Pregnancy and Childbirth and Pos Maternal Responsiveness in this Context Depresión en la Gestación y en Posparto y la Capacidad de Respuesta Materna en ese Contexto}

\author{
Adriane Diehl Krob \\ Josehelen de Godoy ${ }^{1}$ \\ Keila Pamela Leite \\ Samantha Gottardo Mori \\ Centro Universitário Dinâmica das Cataratas (UDC)
}

\begin{abstract}
Resumo
O presente artigo visa à explanação da responsividade materna no contexto da depressão pré-natal e no pós-parto. Estudos apontam que a depressão tem impactos negativos na relação mãe-bebê já no período gestacional e mais gravemente no pós-parto. Esses impactos gerados pela depressão interferem na vinculação segura entre a díade, o que prejudica a responsividade materna e, por sua vez, as respostas de apego do bebê à mãe. Dessa forma, compreender como a responsividade materna se desenvolve nesse contexto pode ser fundamental para assegurar a saúde da díade e, consequentemente, de toda a família. Portanto é essencial que o acompanhamento às gestantes passe a ter um olhar mais humanizado pelos profissionais da saúde através de uma equipe multidisciplinar, que trabalhe não só com os aspectos relacionados à saúde mãe-bebê, mas, inclusive, com os aspectos emocionais da gestante, auxiliando assim na detecção ou prevenção da depressão na gestação e no pós-parto.

Palavras-chave: responsividade materna, depressão gestacional, depressão pós-parto, interação mãe-bebê
\end{abstract}

\begin{abstract}
This article aims at the explanation of maternal responsiveness in the context of antenatal depression and postpartum. Studies show that depression has major negative impacts on the mother-baby in the gestational period and more serious postpartum. These impacts generated by depression interfere with secure attachment between the dyad which impairs maternal responsiveness and in turn the responses of infant attachment to the mother. This way, understanding how maternal responsiveness develops in this context, it can be critical to ensure the health of the mother, baby and therefore the whole family. So it is essential that monitoring pregnant women be more humanized by health professionals through a multidisciplinary team, working not only with aspects related to mother-child health, but also with the emotional aspects of the pregnant woman, thus aiding in the detection or prevention of depression during pregnancy and postpartum.

Keywords: maternal responsiveness, depression in pregnancy, depression in childbirth, mother-infant interaction

\section{Resumen}

Este artículo tiene como objetivo explicar la capacidad de respuesta materna en el contexto de la depresión prenatal y posparto. Estudios muestran que la depresión tiene repercusiones negativas en la relación madre-hijo ya durante el embarazo y aún más gravemente después del parto. Esos impactos causados por la depresión interfieren en el vínculo seguro entre la díada, lo que deteriora la capacidad de respuesta de la madre y, a su vez, la del bebé de apego a la madre. Así que la comprensión de cómo se desarrolla la capacidad de respuesta materna en ese contexto puede ser fundamental para garantizar la salud de la díada y por lo tanto de toda la familia. Entonces, es esencial que el seguimiento de las mujeres embarazadas tengan un aspecto más humanizado por profesionales de la salud mediante un equipo multidisciplinario, trabajando no sólo con los aspectos relacionados con la salud materno-infantil, pero incluso con los aspectos emocionales de la mujer embarazada, ayudando así en la detección o prevención de la depresión durante el embarazo y después del parto.

Palabras clave: capacidad de respuesta materna, depresión gestacional, depresión posparto, interacción madre-bebé
\end{abstract}

${ }^{1}$ Endereço de contato: Centro Universitário Dinâmica das Cataratas- UDC, unidade Vila A. Av. Paraná, 5661 Vila A, Foz do Iguaçu, PR, Brasil. CEP 85868-030. E-mail: josi_godoy_@hotmail.com 


\section{Introdução}

Muitas mulheres sonham em engravidar e têm muitas expectativas quanto a essa fase, até mesmo a crença popular é de que a maternidade é um período de muita alegria para a mulher. E, por muito tempo, acreditou-se que, nesse período, a mulher estava imune a qualquer doença mental, embora pesquisas recentes apontem riscos psicológicos para essa fase da vida.

A gestação e o pós-parto é um período de muitas adaptações na vida da mulher, pois ocorrem inúmeras mudanças em seu corpo, alterando seu metabolismo e a produção de hormônios. É, também, um momento de reformulação de seu papel social e de alteração da sua psique, afinal marca uma importante etapa da vida. Consiste na criação de um novo sujeito, que se desenvolve durante nove meses, esperado geralmente com muita ansiedade. Além disso, há a construção social de um ideal de ser mãe, o qual muitas gestantes esperam atingir. Tudo isso faz com que se elevem os riscos de desenvolver alterações psicológicas nesse período, até mesmo desencadear transtornos de humor (Baptista, Baptista, \& Oliveira, 2004).

Sabe-se que o estresse emocional presente no período da gravidez tem apontado consequências prejudiciais sobre o embrião. Ademais, a depressão e ansiedade na gestação parecem estar diretamente ligadas à depressão após o parto (DPP). Então, na DPP, a interação entre mãe e filho pode ser comprometida, o que prejudica ainda mais o desenvolvimento do recém-nascido. A mãe deprimida não tem estabilidade emocional suficiente para interagir adequadamente com seu bebê. Esta tende a responder menos aos comportamentos do bebê do que mães não deprimidas, e filhos de mães não responsivas apresentam maiores dificuldades no estabelecimento de apego (Schwengber \& Piccinini, 2003).

Dessa maneira, faz-se cada vez mais necessário averiguar a depressão na gestação e no pós- parto. Assim, identificar e tratar transtornos de humor o quanto antes pode ser o diferencial para garantir a saúde da mãe, do bebê e, por conseguinte, de toda a família. Para a área de conhecimento da Psicologia, desenvolver uma pesquisa sobre esse assunto, poderá posteriormente servir de base para intervenção.

A partir disso, o presente artigo trata-se de uma pesquisa bibliográfica, em bancos de teses e dissertações, bibliotecas eletrônicas como Scielo, Bireme, NCBI, livros e revistas científicas, utilizando para a pesquisa os seguintes termos: depressão/na gestação/na gravidez/no pós- parto/puérperas, responsividade materna, interação mãe-bebê na gestação. A pesquisa teve por objetivo descrever a responsividade materna relacionada com a sintomatologia da depressão na gestação e no pós-parto, bem como, através da literatura, compreender o efeito destas sobre o desenvolvimento do bebê.

\section{A Interação Mãe-Bebê}

A relação mãe-bebê inicia-se no período gestacional, pois, segundo Loreto (2008), é através da placenta que a gestante e o feto se comunicam, e, além de transferir substâncias nutritivas do sangue da mãe para o bebê e alguns hormônios essenciais nessa fase, a placenta transfere também a comunicação afetiva que essa mãe tem para com o feto. De acordo com estudiosos (Piccinini, Gomes, Moreira, \& Lopes, 2004), a relação mãe-bebê começa no período pré-natal, e essa relação tende a ser muito intensa, já que está apoiada nas expectativas 
da gestante sobre o bebê. Já no início da gestação, a mãe consegue ter uma interação com o feto, através do descobrimento do sexo e da maneira de movimentar-se desse bebê. Muitas pesquisam apontam que, quando a mãe interage com o feto, conversando e pensando nele, ajuda na construção da relação mãe-bebê posteriormente, já que, para estabelecer este vínculo, é necessário que essa mãe tenha uma boa vivência de suas experiências da gestação e do puerpério (Borsa \& Dias, 2004). No entanto, para esses autores, aquela mãe que não se sente preparada para o nascimento do bebê e os posteriores cuidados com ele e ainda não encontra rede de apoio na gestação, poderá sentir-se inapropriada, e a vinculação da mãe para com o filho poderá ser mais difícil.

Durante o período gestacional, a mulher passa por várias mudanças, tanto físicas como emocionais e hormonais. E essas mudanças, segundo Maldonado (2002), envolvem prós e contras, perdas e ganhos, o que explicaria os sentimentos opostos que essas gestantes possuem em relação ao bebê e à gestação em si. Ainda segundo esta autora, essa ambivalência de sentimentos muda com o nascimento do bebê, pois a realidade do feto difere da realidade do recém-nascido, já que este é mais um novo membro da família e que, consequentemente acaba mudando o equilíbrio familiar. Por isso muitas mães têm dificuldade em aceitar tal realidade, ficando assustadas diante dos primeiros cuidados com o filho.

Segundo Borsa e Dias (2004), a história de vida da mãe poderá influenciar o seu sentimento e o seu comportamento em relação a seu bebê; o seu padrão de relacionamento parental e pessoal que construiu ao longo da vida é que dará origem à maneira como essa mãe irá se comportar e se relacionar com a criança. O comportamento da mãe em relação ao bebê e o vínculo que esta díade criar darão respaldo às relações que a criança irá formar ao longo da vida.

\section{Depressão}

A depressão Maior é abrangente, abarca possivelmente uma gama muito heterogênea de condições, que vão desde as fronteiras da normalidade (reações de luto ou tristeza normal) até aquelas formas mais graves de depressão, para as quais provavelmente concorrem fatores biológicos - adquiridos e/ou geneticamente determinados (Cruz, Simoes, \& Faisal-Cury, 2005).

A depressão é um transtorno que essencialmente se manifesta por episódios depressivos recorrentes, ou seja, os episódios podem durar meses a anos, porém, se não tratados, podem levar a um curso crônico, de acordo com o DSM-IV-TR (American Psychiatric Association, 2002). O episódio de depressão pode ser classificado em leve, moderado ou severo, de acordo com a gravidade ou a quantidade dos sintomas. Para o diagnóstico de "Transtorno Depressivo Maior", é necessário que a pessoa apresente "humor deprimido ou perda de interesse ou prazer, durante um período de duas semanas", mais quatro sintomas de uma lista de nove (ou mais três sintomas, se os dois primeiros estiverem presentes). Os sintomas da depressão são humor deprimido, anedonia, alterações de apetite, peso e sono, variação da psicomotricidade, fadiga ou perda de energia, culpa e inutilidade, dificuldades de concentração e tomada de decisões, pensamentos de morte incluindo ideação suicida, planos e tentativas de suicídio.

As mulheres têm mais predisposição do que homens para desenvolver a depressão, a relação entre homens e mulheres é aproximadamente de 2:1 (Barlow \& Durand, 2011). 
Estima-se que o risco de desenvolver depressão, ao longo da vida, seja de 10\% para os homens e de 20\% para as mulheres (Cruz et al., 2005). Algumas razões foram apresentadas por Holmes (1997) para a prevalência de depressão no sexo feminino, dentre as quais: a) as mulheres expressam seus sentimentos de maneira mais livre devido a seus papéis sociais, consequentemente os sentimentos associados à depressão também, b) são expostas a situações mais estressantes, c) questões hormonais e fisiológicas predispõem as mulheres a terem mais depressão que os homens.

\section{Depressão na Gestação}

A depressão pode surgir a qualquer momento da vida da mulher, inclusive no período gestacional. Isso ocorre muito embora haja uma crença social de que esse período proporcione mais união ao casal e que seja uma etapa de alegria, no entanto o período perinatal em nada protege a mulher de transtornos de humor (Pereira, Lovisi, Lima, \& Legay, 2010). Isto ocorre principalmente na sociedade de hoje, onde a mulher muitas vezes encontra-se desamparada: ela precisa lidar com muitas questões (Loreto, 2008), que incluem mudanças corporais, psíquicas, hormonais, além de ter uma vida social, trabalho, afazeres domésticos, cuidados com outros filhos. Por isso tudo, esse período se torna um "tsunami" de emoções desencadeadas pela maternidade e, muitas vezes, desperta na mulher emocionalmente vulnerável alguns sentimentos como angústia e depressão. No período gestacional, a muIher deve aceitar as modificações físicas da gravidez, sua nova imagem corporal, as novas configurações de relacionamento com o companheiro e sua família, a chegada de um novo membro e a sua nova identidade pessoal; contudo perturbações depressivas nessa fase fazem com que a mãe tenha dificuldade em lidar com essas questões (Pereira et al., 2010).

São diversas as emoções com que a mulher deve lidar nesse período. Tedesco, Quayle, e Zugaib (1997) listam desde alterações de humor, passividade e inquietude à depressão, decorrentes da ansiedade instalada. Autores salientam que a depressão pode estar diretamente associada à maternidade, pois é nesse período que os níveis de serotonina diminuem junto com os fatores estressantes do período gestacional (Baptista et al., 2004). Além disso, segundo o DSM-IV-TR (American Psychiatric Associatoin, 2002), sintomas depressivos apresentados no período gestacional bem como a tristeza pós-parto, conhecida como "baby blues", aumentam o risco de depressão no pós-parto. Um estudo de Murata, Lima, Bonadio, e Tsunechiro (2012) concluiu que muitas grávidas apresentam sintomas depressivos maiores, e enfatizou a extrema necessidade de observar a saúde mental delas já no início da gestação, primando a prevenção de transtornos de humor de modo a evitar possíveis transtornos no período perinatal e, caso estes já estejam presentes, melhorá-los. Um estudo de Pereira e Lovisi (2008) analisou de maneira geral a média da prevalência de depressão em gestantes e constatou que esta é em torno de $20 \%$ em países subdesenvolvidos, inclusive no Brasil, e de 10\% e 15\% aproximadamente, nos países desenvolvidos. Um estudo de Bennett, Einarson, Taddio, Koren e Einarson (2004), cujo objetivo foi estimar a prevalência de depressão durante a gravidez por trimestre, constatou que $7,4 \%$ das gestantes apresentaram sintomas depressivos no primeiro trimestre de gestação; no segundo, a prevalência foi de $12,8 \%$, e $12 \%$ no terceiro trimestre, concluindo assim que as taxas de depressão, especialmente durante o segundo e terceiro trimestres da gravidez, são essenciais para o diagnóstico e tratamento 
de depressão maior. Cerca de um quinto das mulheres no período gestacional apresentam depressão, porém a maioria delas não é tratada (Pereira \& Lovisi, 2008). Gestantes com quadros depressivos não tratados durante a gravidez tendem a se expor ao álcool e outras drogas, apresentam falta de apetite, desnutrição, dificuldades de aceitarem recomendações médicas e não continuação do pré-natal, com consequente risco de mortalidade neonatal (Pereira et al., 2010).

A depressão pré-natal, segundo a literatura, tem sido negligenciada e não levada muito a sério, porém ela é fator de risco para a depressão pós-natal e para o desenvolvimento da criança (Pereira \& Lovisi, 2008). Estudos apontam que, na gestação, a mãe e o feto interagem de maneira que tudo que a mãe sente, ingere e pensa afeta diretamente o feto no período pré e pós-gestacional (Loreto, 2008). Assim como Payne (in Felix, Gomes, e França, 2008) afirma, a depressão da mãe após o nascimento do filho pode acarretar em descuido e até abusos ao recém-nato, já que este demonstra muita necessidade de cuidado em seus primeiros anos de vida. Isso danifica o vínculo mãe-bebê e tem como consequências para o infante insegurança, desajustamento emocional, problemas cognitivos, de linguagem e atenção.

\section{Depressão Pós-Parto}

O parto é um período desencadeador de inúmeras mudanças intra e interpessoais, e, após o nascimento do bebê, essas mudanças aceleram-se tanto na família, como na mulher.

Dentre os transtornos depressivos que acometem mulheres, está a depressão pós-parto que atinge cerca de 6,8 a 16,5\% das mulheres adultas e até $26 \%$ em adolescentes. A DPP não se difere da sintomatologia dos episódios de alterações de humor que ocorrem fora do período gestacional (Cruz et al., 2005). A DPP é um dos três distúrbios de humor que acomete as mulheres após o parto, o mais leve deles é o Baby blues conhecido também como tristeza pós- parto ou melancolia da maternidade (Cantilino, Zambaldi, Sougey, \& Renno, 2010). Segundo Kaplan e Sadock (1990), esse distúrbio pode ser caracterizada pela alteração de humor que acomete cerca de $50 \%$ das puérperas entre o terceiro e o quinto dia após o parto, e que geralmente some com os passar dos dias. Essa alteração de humor consiste em sentimentos de tristeza, choro, angústia e irritabilidade, e ocorre devido às mudanças hormonais, ao estresse durante o parto, e ao aumento da responsabilidade e expectativas frente à maternidade.

Contudo outras podem apresentar depressões mais graves que podem causar incapacidade para o autocuidado e para o cuidado dos filhos, como, por exemplo, a Psicose pós-parto, sendo considerada a mais grave dentre as três, e que acomete 0,1\% das mulheres que acabaram de dar à luz, seus sintomas aparecem também nos primeiros dias ou até duas semanas após o nascimento do bebê (Cantilino et al., 2010). Ainda segundo esses autores, os primeiros sintomas são euforia, irritabilidade e perda do sono, para logo em seguida surgirem sintomas mais graves como delírios, alucinações, confusão mental e surtos psicóticos, sendo a psicose pós-parto a grande causa do infanticídio. Já a depressão pós-parto refere-se ao conjunto de sintomas, geralmente iniciados entre a quarta e oitava semana após o parto, alguns sintomas são descritos pelo autor como irritabilidade, choro, sentimento de desamparo e desesperança, falta de energia e motivação, desinteresse sexual, transtor- 
nos alimentares e do sono, incapacidade de lidar com novas situações e queixas psicossomáticas (Klaus, Kennel, \& Klaus, 2000). De acordo com o DSM-IV-TR (American Psychiatric Association, 2002), os comportamentos das mães com depressão pós-parto variam desde o desinteresse e medo de ficar a sós com o bebê, como também o excesso de cuidado ao bebê, dificultando o repouso adequado dele, prejudicando-o.

Alguns estudos de Schwengber e Piccinini (2003) indicam associação entre a ocorrência da DPP relacionada ao pouco suporte oferecido pelo parceiro ou por outras pessoas que mantêm relacionamento. Isso é complementado em um estudo feito por Cruz et al. (2005), realizado a partir de dados obtidos do Programa Saúde da Família, sobre a DPP, o qual concluiu a presença do marido como sendo suporte social, e um importante fator preventivo na presença do transtorno. O não planejamento da gestação, o nascimento prematuro e a morte do bebê, a dificuldade em amamentar e as dificuldades no parto são alguns fatores causais da DPP (Schwengber \& Piccinini, 2003). Os fatores de riscos identificados por um estudo de Silva, Araújo, Araújo, Carvalho, e Caetano (2010) relacionados à DPP foram: sintoma depressivo durante a gravidez, histórico anterior à gestação de depressão, autoestima limitada, problemas conjugais, ausência de parceiro e instabilidade financeira. Além do mais, as consequências da DPP se estendem aos aspectos emocional, social e cognitivo da criança (Schmidt, Piccoloto, \& Müller, 2005).

É essencial, conforme Falcone, Mäder, Nascimento, Santos, e Nóbrega (2005), que o atendimento as gestantes por parte dos profissionais de saúde seja humanizado, propiciando a família um cuidado que valorize os aspectos inconscientes da maternagem, como as emoções e experiências do período. Isso pode proporcionar desde a detecção da DPP, como a prevenção desta. Nesse sentido, Tostes (2012) sugere o Pré-Natal Psicológico (PNP), diferentemente dos cursos de gestantes e raramente encontrado em serviços de obstetrícia. Trata-se de uma modalidade de atendimento perinatal, voltada para maior humanização do processo gestacional do parto e da construção da parentalidade.

É de suma importância a identificação precisa do episódio de DPP, a qual deve ser tratada em uma abordagem multidisciplinar, com tratamentos específicos, uma vez que essa patologia pode ser muito prejudicial, tanto física quanto psiquicamente. Pois, "é necessário que esta experiência vivida subjetivamente pela mulher, possa ser detectada para ajudá-la no processo de reconstrução" (Schmidt et al., 2005).

Para o tratamento da DPP, a utilização de medicamentos antidepressivos têm se mostrado o primeiro recurso para o tratamento, embora ocasione prejuízos no aleitamento materno e poucas pesquisas tenham sido realizadas. Outro recurso, muito verificado que se mostrou eficaz foi a terapia cognitivo-comportamental- TCC (Cantilino et al., 2010).

\section{Responsividade Materna}

Na interação mãe-bebê, a responsividade materna "é um elemento crítico desse sistema, visto que o adulto é o responsável pelo estabelecimento das interações do bebê com o meio ambiente, devendo conduzir esse processo de maneira adaptada às habilidades da criança" (Lordelo, Fonseca, \& Araujo, 2000, p. 74).

De acordo com Ribas e Moura (2004), embora se utilizem diversificadas abordagens quanto ao tema, discute-se este principalmente dentro do processo de interação mãe-filho 
e quanto à validade preditiva da responsividade materna sobre o desenvolvimento infantil (desenvolvimento da cognição, linguagem, emoção e sociabilidade). A validade preditiva refere-se ao que afirmam Piccinini, Marin, Alvarenga, Lopes, e Tudge (2007) de que a responsividade da mãe com seu bebê, avaliada no início da vida deste, pode ser um excelente previdente de características do desenvolvimento da criança, isto é, tem relação com as capacidades cognitivas e de atenção que a criança apresentará posteriormente. Assim como, de acordo com Chalhub (2004), a responsividade da mãe tem influência em todas as futuras interações que a criança apresentará.

O termo responsividade tem uma variedade de conceituações e tem sido foco de diversos pesquisadores, embora o conceito tenha sido pouco discutido no Brasil, o que não permite comparações com a conceituação internacional (Lordelo, 2002; Ribas \& Moura; 2004). O conceito de responsividade materna está intimamente ligado às teorias de vinculação, dentre elas, a formulada por Bowlby (1984), do apego, e a teoria de Winnicott (1999) sobre o conceito da mãe suficientemente boa. De acordo com a teoria de Bowlby (1984), apego é uma forma de vinculação, em que a pessoa se sente segura e amparada na presença da figura de apego; no caso dos bebês, essa figura de apego é um adulto, geralmente as mães, isso, pois, a necessidade de cuidado e sobrevivência das crianças depende da disponibilidade do outro, ou seja, de um ser adulto que demonstre segurança, conforto e o alimente. $\mathrm{O}$ estabelecimento de vínculos é algo essencial para o desenvolvimento da natureza humana, esses vínculos tornam- se laços duradouros estabelecidos entre a díade. A demonstração do apego é vista através de comportamentos observáveis e organizados.

O conceito responsividade materna tem sido utilizado "como variedade de respostas maternas sobre uma variedade de sinais infantis" (Chalhub, 2004). Wakschlag e Hans (in Piccinini et al., 2007) definem responsividade materna como comportamentos maternos esperáveis para a compreensão da interação mãe-bebê, os quais são necessários para o desenvolvimento do apego e interação mútua entre a díade, enquanto Bornstein e TamisLeMonda (1997) definem responsividade como comportamentos maternos contingentes, apropriados e imediatamente relacionados aos comportamentos das crianças. Chalhub (2004) identifica duas características principais da responsividade da mãe: a qualitativa, que considera aspectos afetivos; e a temporal a qual se refere às contingências de respostas. Há também o conceito de sensitividade, semelhante à responsividade, definido pelos autores como "as qualificações do tipo calor, proximidade, intimidade, que seriam dimensões avaliativas mais qualitativas e afetivas, quanto à dimensão temporal da contingência da resposta" (Ribas \& Moura, 2004).

A responsividade materna é observada nas seguintes categorias observáveis: "iniciativa de comunicação, exclusividade de comunicação, contato físico, conteúdo da comunicação, natureza afetiva (tom emocional) e estilos de cuidado e ensino" (Chalhub, 2004). Para o autor a responsividade materna faz parte de um diálogo presente na relação mãe bebê e envolve duas competências necessárias à mãe, a capacidade de interpretação das necessidades do filho e a adequação das respostas. As respostas não adequadas podem ser vistas como negligências ou ainda protecionismo. Já para Eshel, Daelmans, Mello, e Martines (2006), três etapas são envolvidas no processo de responsividade: a primeira é a observação, na qual o cuidador observa os sinais da criança, desde movimentos a vocalizações; a segunda é referente à interpretação dos sinais, se essa interpretação é correta; e a última refere-se à ação 
tomada pelo cuidador, se este age rapidamente, se é consistente e eficaz. Essa resposta da interação por parte do cuidador, se contingente e adequada, é vital para a criança e garante um melhor desenvolvimento cognitivo e psicossocial.

A responsividade materna é avaliada de acordo com a sincronia interacional. A sincronia evidencia a experiência da interação mútua que demonstra a adaptação dos comportamentos da mãe concomitantemente com o do bebê. Essa sincronia, segundo os autores, ocorre "através de categorização de coocorrências de comportamento da mãe e da criança relacionadas temporalmente, classificadas como sincrônica ou assincrônicas" (Piccinini \& Seidl-de-Moura, 2007). Quando a responsividade materna é adequada, a mãe busca ajustar seu comportamento ao do bebê, estimulando-o. Dessa forma, as respostas de afetividade tornam-se sincrônicas e a interação é mutua; porém, quando a mãe se torna indisposta a responder emocionalmente ao bebê, tais comportamentos tornam-se assincrônicos, pois a criança perde o referencial externo de estimulação (Field, 1995).

Existem métodos de mensuração da responsividade materna, os dois apresentados a seguir se utilizam de observação e filmagem da interação mãe-bebê, em que a mãe interage naturalmente com o seu bebê por um período de tempo. O primeiro se utiliza da Escala de Avaliação da Responsividade Materna (EARM), descrito por Landry, Miller-Loncar, e Swank (in Ferreira \& Abreu-Lima, 2008) e consiste em um protocolo de observação, usado durante os comportamentos maternos na interação da díade, com duração de 15 minutos o qual descreve oito componentes de responsividade: "manifestação de afeto positivo", "calor afetivo", "flexibilidade/responsividade", "intrusão física", "negatividade", "demonstração ensino físico", "conteúdo verbal", "suporte verbal". Nesse instrumento, segundo Ferreira e AbreuLima (2008), duas escalas são utilizadas para avaliação da responsividade materna, a primeira em que "as cotações mais elevadas se baseiam no facto da mãe e da criança se comportarem ou demonstrarem comportamentos que vão de encontro a um conjunto de critérios de cotação" (p. 13), ou seja, cada componente é cotado através de uma escala tipo Likert de 5 pontos, sendo que os valores mais elevados indicam mais responsividade por parte das mães, para ser confiada a pontuação 5 no item "Elevado calor afectivo" no componente "Calor afectivo" todos os critérios listados devem ser observados "(envolvimento, proximidade física, verbalizações positivas, entusiasmo, afecto físico, aceitação, alegria/prazer, na interação encorajamentos e elogios, manipulação e reposicionamento sensíveis ao estilo de interação relaxado)". A segunda escala tem como foco a proporção de tempo em que a mãe responde através de comportamentos ao bebê; para exemplificar, na escala "Demonstração/ ensino físico", a pontuação máxima só é dada se a mãe mantiver, na maior parte do tempo da observação, respostas comportamentais de demonstração de "cariz instrutiva e interactiva, orientada pela criança e com recursos a estratégias do tipo mão sobre mão". Segundo instrumento de análise utilizado por estudos sobre responsividade materna é o método do GIDEP (Grupo de Pesquisa em Infância, Desenvolvimento e Psicopatologia), utilizado no Brasil, em Porto Alegre, RS, e que consiste também na filmagem da interação mãe e bebê. Após filmar a interação mãe-bebê, as imagens são analisadas considerando "dois aspectos do comportamento materno em relação ao comportamento da criança: adequação e contingência" (Piccinini et al., 2007). A adequação refere-se ao tipo de resposta apresentada pela mãe, se são comportamentos facilitadores, como demonstração física e verbal de afeto, e a contingência é em relação ao tempo, respostas da mãe que ocorrem após 12 segundos de- 
pois do comportamento do bebê. As respostas das mães que foram adequadas aos comportamentos do bebê são comportamentos maternos facilitadores da interação; pode-se citar alguns como falar com o bebê, embalar, estimular, sorrir, afagar, pegar no colo (Piccinini et al., 2007). Inicialmente o primeiro e o último minuto da filmagem são descartados, e seis minutos da filmagem são selecionados para análise, os três minutos iniciais e os três finais, os quais são divididos em intervalos de 12 segundos. Nos seis primeiros segundos, os comportamentos do bebê são analisados em categorias: O bebê sorri visivelmente, o bebê vocaliza, chora. As respostas da mãe a esses comportamentos do bebê serão registradas no mesmo intervalo e no intervalo seguinte em categorias: A mãe interpreta/fala pelo bebê, fala para o bebê, sorri para o bebê, pega no colo/embala, beija/acaricia, toca ou estimula com um objeto, oferece chupeta/seio/mamadeira. Após os primeiros três minutos do total de seis minutos, é realizada a mesma observação com as mesmas categorias nos minutos restantes. Dessa forma, são analisadas as sequências responsivas e não-responsivas, considerando-se os critérios de contingência e adequação da resposta materna; após essa análise, as concordâncias das categorias são calculadas por meio de instrumentos como o coeficiente Kappa, que pode ser definido como uma medida de associação usada para descrever e testar o grau de concordância (confiabilidade e precisão) na classificação. "Para cada uma das sequências responsivas foi calculado o percentual de responsividade materna dividindo-se o número de ocorrências naquela sequência pelo número de comportamentos do bebê em questão" (Piccinini et al., 2007). O mesmo procedimento é adotado para se obter os percentuais nas sequências não responsivas.

Nessa lógica em que os comportamentos são observáveis, é necessário, de acordo com Ribas e Moura (2004), considerar em pesquisas sobre responsividade e os aspectos maternos, compreender as práticas culturais envolvidas nos cuidados com crianças. Para Lordelo (2002), quando se investiga a responsividade, há uma tendência a enquadrá-la como um fator de personalidade da mãe, em detrimento aos aspectos culturais no que se refere ao trato com crianças. A autora entende o conceito de responsividade como algo inerente ao ser humano, de caráter biológico, o qual tem a função de garantir a sobrevivência dos bebês, somada as características sociais, as quais dependem do período histórico vivenciado, a classe social pertencente e imposições culturais. "A responsividade pode depender de numerosos fatores do ambiente, incluindo as concepções dos indivíduos sobre maternidade, filhos e sistemas de criação, dependentes, em última análise, das ideias e práticas culturais compartilhadas por um grupo social" (Lordelo et al., 2000). É preciso considerar também fatores ambientais como o raciocínio pessoal/cognição, linguístico, socioemocional, compreendendo suas particularidades no que tange a temperamento, submissão, ansiedade, stress, abuso de drogas e depressão (Ribas \& Moura, 2004).

Os bebês são mais vulneráveis ao impacto da DPP. A qualidade da interação mãe-bebê depende principalmente da responsividade emocional da mãe. Desse modo, quanto mais grave for a depressão materna, maior será o impacto negativo na relação da mãe com o bebê, bem como o desenvolvimento da própria criança (Cantilino et al., 2010). Stern (1997) acrescenta que, quando a mãe está deprimida, um dos seus primeiros eventos comportamentais é romper com seu bebê o contato visual, e, portanto acaba tornando-se menos responsiva, demonstrando apatia e falta de ânimo, e isso afeta o bebê: este vivencia a depressão da mãe em seus microeventos e Ihes atribui significância. Os microeventos, segundo o autor, são 
pequenas manifestações, como por exemplo, quando o bebê sorri e a mãe retribui sorrindo a ele. No caso de resposta não adequada, o bebê vai, aos poucos, se afastando de sua mãe, começa a apresentar desânimo, postura deprimida, diminui suas demonstrações de afetividade e expressividades faciais. Portanto o bebê, ao não receber as respostas da mãe mediante aos seus comportamentos, desenvolve um jeito depressivo de interação (Field, 2002).

A DPP influencia negativamente nos comportamentos afetivos da interação mãe-bebê, até a interação tornar-se assincrônica; o bebê diminui sua contingência de respostas por compreender que seu comportamento não tem influência no comportamento materno, assim, o bebê passa a não responder à mãe, conforme Field, Healy, Goldstein, e Guthertz (in Carlesso, 2011). Ainda de acordo com os autores, pode ser que essa mãe apresente frustração com a diminuição das ações da criança e passe a utilizar de comportamentos intrusivos com intuito de obter respostas. Field (2002) esclarece que, nesse contexto, existem dois tipos de interacionalidade de mães com seus bebês, o primeiro modelo é o intrusivo, mães que estimulam de maneira excessiva sua criança, e o outro modelo é de mães com interações apáticas. Ambos os modelos são prejudiciais às crianças, pois os bebês respondem a essas mães através de interações apáticas, depressivas, com dificuldades psicomotoras, irritabilidade, nível exacerbado de atividades interacionais. Nesse sentido, a depressão materna influencia seriamente na interação mãe-bebê, ainda mais quando não há alguém que substitua a função materna comprometida pela DPP.

Spitz (1979) trata a depressão pós-parto como uma ruptura grave na relação mãe-bebê, na qual o bebê sente a perda da mãe não no aspecto físico, uma vez que ela está presente, essa perda é diferente de um processo de luto; trata-se de um distanciamento emocional da mãe para com o seu bebê, ocasionando falta de identificação da mãe como objeto bom.

Desse modo, é importante estabelecer o vínculo da mãe e filho ainda no ventre, uma vez que "um bom vínculo mãe-feto constitui a melhor proteção contra os perigos do mundo exterior, e seus efeitos não são limitados ao período intra-uterino" (Falcone et al., 2005, p. 5). Esses autores sugerem como estratégia o fortalecimento do vínculo nesse período através de cantigas que a mãe ofereça a seu bebê.

Um estudo verificou que quanto mais instruídas as mães, maior a atenção da mãe em compreender os sinais do filho e, consequentemente, maior a tentativa de interação com a criança, "sublinhando a importância da educação materna para que as tarefas ligadas à parentalidade sejam desempenhadas de maneira satisfatória, de modo a favorecer o desenvolvimento infantil pleno nos primeiros anos de vida" (Alvarenga, Malhado, \& Lins, 2014, p. 312).

\section{Conclusão}

De acordo com o referencial bibliográfico revisado, foi possível verificar como os impactos da depressão no contexto gestacional e no pós-parto influenciam na interação da díade. Sabe-se que a responsividade materna inicia-se desde a gestação com os primeiros sinais que o bebê emite dentro do ventre da mãe e as respostas que essa infere sobre esse bebê, sendo as vocalizações os primeiros sinais de responsividade materna. Porém algumas muIheres, devido a uma série de fatores como, alterações hormonais, expectativas em relação à nova etapa de vida, crenças sociais sobre a maternidade e dificuldades no âmbito familiar e na rede de apoio, desenvolvem um quadro depressivo, trazendo a essa mãe algumas dificuldades de interação e aceitação do bebê. 
Estudos apontam que a prevalência ou aumento dos sintomas depressivos no período pós-parto, repercutem de forma negativa nas interações iniciais mãe-bebê, causando na mãe, inabilidade de cuidados a criança e falta de sensitividade aos comportamentos desta, afetando o desenvolvimento cognitivo, emocional e social do bebê. Além disso, observa-se que interação mãe-bebê ocorre mediante a criação de apego seguro e estabelecimento de vínculos. Quando esses aspectos são desenvolvidos adequadamente, a responsividade materna se torna saudável, e a mãe prontamente atende as solicitações comportamentais emitidas pelo filho. Já no contexto da depressão, a mãe não consegue estabelecer o vínculo seguro e adequado com a criança; dessa forma, rompe o contato materno e torna-se insensível aos apelos do bebê.

Nesse sentido, são necessárias avaliações sobre a depressão desde a gestação; por isso instrumentos e intervenções apropriadas para essa patologia nesse período auxiliariam na prevenção dessas dificuldades apresentadas pelas mães na interação com o bebê. Da mesma forma, o diagnóstico da DPP já realizado após o nascimento do bebê representa para a área de saúde um desafio a ser pesquisado, já que o conceito de responsividade materna é algo novo no Brasil, e poucos são os estudos realizados no país a respeito dessa forma de verificação de interação materna.

\section{Referências}

Alvarenga, P., Malhado, S. C. B., \& Lins, T. C. S. (2014). O impacto da responsividade materna aos oito meses da criança sobre as práticas de socialização maternas aos 18 meses. Estudos de Psicologia, 19(4), 305-314. Disponível em http://dx.doi.org/10.1590/S1413 $-294 \times 2014000400008$

American Psychiatric Association. (2002). DSM-IV-TR - Manual diagnóstico e estatístico de transtornos mentais (4a ed. rev.). Porto Alegre: Artmed.

Baptista, M. N., Baptista, A. S. D. E., \& Oliveira, M. G. (2004). Depressão e gênero: Por que as mulheres se deprimem mais que os homens? Temas Psicologia, 7(2), 143-156. Disponível em http://pepsic.bvsalud.org/scielo.php?script=sci_arttext\&pid=S1413-389X 1999000200005\&lng=pt\&nrm=iso

Barlow, D. H., \& Durand, R. M. (2011). Psicopatologia: Uma abordagem integrada. São Paulo: Cengage Learning.

Bennett, H. A., Einarson, A., Taddio, A., Koren, G., \& Einarson, T. R. (2004). Prevalence of depression during pregnancy: systematic review. Obstetrics and Gynecology, 103(4), 698-709.

Bornstein, M. H., \& Tamis-LeMonda, C. S. (1997). Maternal responsiveness and infant mental abilities: Specific predictive relations. Infant Behavior and Development, 20(3), 283-296.

Borsa, J. C., \& Dias, A. C. G. (2004). Relação mãe e bebê: As expectativas e vivências do puerpério. Revista Perspectiva, 28(102), 39-53.

Bowlby, J. (1984). Apego - Apego e perda (Vol. 1). São Paulo: Martins Fontes.

Cantilino, A., Zambaldi, C. F., Sougey, E. B., \& Renno Jr., J. (2010). Transtornos psiquiátricos no pós-parto. Revista de Psiquiatria Clínica, 37(6), 288-294 . Disponível em http://www.scielo.br/scielo.php?script=sci_arttext\&pid=S0101-60832010000600006\&lng=en\&nrm=iso. ISSN 0101-6083 
Carlesso, J. P. P. (2011). Análise da relação entre depressão materna e índices de risco ao desenvolvimento infantil (Dissertação de Mestrado, Universidade Federal de Santa Maria, RS).

Chalhub, A. A. (2004). Padrões comportamentais maternos e desenvolvimento cognitivo de pré-escolares em contexto urbano pobre (Dissertação de Mestrado em Psicologia, Faculdade de Filosofia e Ciências Humanas, Universidade Federal da Bahia, Salvador, Bahia).

Cruz, E. B. S., Simoes, G. L., \& Faisal-Cury, A. (2005). Rastreamento da depressão pós-parto em mulheres atendidas pelo Programa de Saúde da Família. Revista Brasileira de Ginecologia e Obstetrícia [online], 27(4), 181-188. Disponível em www.scielo.br/pdf/rbgo/v27n4/ a04v27n4.pdf

Eshel, N., Daelmans, B., Mello, M. C., \& Martines, J. (2006). Responsive parenting: Interventions and outcomes. Bull World Health Organ, 84(12), 991-998. Disponível em https://www.ncbi.nlm.nih.gov/pmc/articles/PMC2627571/pdf/17242836.pdf

Falcone, V. M., Mäder, C. V. N., Nascimento, C. F. L., Santos, J. M. M., \& Nóbrega, F. J. (2005). Atuação multiprofissional e a saúde mental de gestantes. Revista de Saúde Pública, 39(4), 612-618. Disponível em https://dx.doi.org/10.1590/S0034-89102005000400015

Felix, G. M. A., Gomes, A. P. R., \& França, P. S. (2008). Depressão no ciclo gravídico-puerperal. Comunicação em Ciências da Saúde, 19(1), p. 51-60. Disponível em http://www.escs.edu. br/pesquisa/revista/2008Vol19_1art06depressaonocilco.pdf

Ferreira, T., \& Abreu-Lima, I. (2008). Responsividade materna: Revisão conceptual e fundamentos para avaliação. Infâncias possíveis, mundos reais: Actas do 1o Congresso Internacional em Estudos da Criança. Braga: Instituto de Estudos da Criança da Universidade do Minho. Disponível em http://hdl.handle.net/10216/62399

Field, T. (1995). Infants of depressed mothers. Infant Behavior and Development, 18(1), 1-13. Field, T. (2002). Prenatal effects of maternal depression. In:S. H. Goodman \&I. H. Gotlib. Children of depressed parents (pp. 59-88). Washington, DC: American PsychologicalAssociation.

Holmes, D. S. (1997). Psicologia dos transtornos mentais. (2a ed.). Porto Alegre: Artes Médicas.

Kaplan, H. I., \& Sadock, B. J. (1990). Compêndio de Psiquiatria (2a ed.). Porto Alegre: Artes Médicas.

Klaus, M. H., Kennel, J. H., \& Klaus, P. (2000). Vínculo: Construindo as bases para um apego seguro e para a independência. Porto Alegre: Artmed.

Lordelo, E. R. (2002). Interação social e responsividade em ambientes doméstico e de creche: cultura e desenvolvimento. Estudos de Psicologia, 7(2), 343-350. Disponível em http://www. scielo.br/scielo.php?script=sci_arttext\&pid=S1413=294-2002000200015X\&lng=en\&tlngpt. 10.1590/S1413-294X2002000200015

Lordelo, E. R., Fonseca, A. L., \& Araujo, M. L. V. B. (2000). Responsividade do ambiente de desenvolvimento: crenças e práticas como sistema cultural de criação de filhos. Psicologia: Reflexão e Crítica, 13(1), 73-80. Disponível em http://www.scielo.br/scielo. php?script=sci_arttext\&pid=S0102-79722000000100009\&lng=en\&nrm=iso

Loreto, V. (2008). Depressão na gravidez: Repercussões no bebê. In: L. M. Atem (Org.). Cuidados no início da vida: Clínica, instituição, pesquisa e metodologia. São Paulo: Casa do Psicólogo. 
Maldonado, M. T. (2002). Psicologia da gravidez - Parto e puerpério (16a ed.). São Paulo: Saraiva.

Murata, M., Lima, M. O. P., Bonadio, I. C., \& Tsunechiro, M. A. (2012). Sintomas depressivos em gestantes abrigadas em uma maternidade social. REME - Revista Mineira de Enfermagem,16(2), 194-200. Disponível em http://bases.bireme.br/cgi-bin/wxislind. exe/iah/online/?IsisScript=iah/iah. $x i s \& s r c=g o o g l e \& b a s e=B D E N F \& l a n g=p \&$ nextAction=lnk\&exprSearch $=22703$ \&indexSearch $=I D$

Pereira, P. K., Lovisi, G. M., Lima, L. A., \& Legay, L. F. (2010). Complicações obstétricas, eventos estressantes, violência e depressão durante a gravidez em adolescentes atendidas em unidade básica de saúde. Revista de Psiquiatria Clínica, 37(5), 216-222. Disponível em http://www.scielo.br/pdf/rpc/v37n5/a06v37n5.pdf

Pereira, P. K., \& Lovisi, G. M. (2008). Prevalência da depressão gestacional e fatores associados. Revista de Psiquiatria Clínica, 35(4), 144-153. Disponível em http://www.scielo.br/ scielo.php?script=sci_arttext\&pid=S0101-60832008000400004

Piccinini, C. A., \& Seidl-de-Moura, M. L. (2007). Observando a interação pais bebê-criança: Diferentes abordagens teóricas e metodológicas. São Paulo: Casa do Psicólogo.

Piccinini, C. A., Gomes, A. G., Moreira, L. E., \& Lopes, R. S. (2004) Expectativas e sentimentos da gestante em relação ao seu bebê. Psicologia: Teoria e Pesquisa, 20(3), 223-232.

Piccinini, C. A., Marin, A. H., Alvarenga, P., Lopes, R. C. S., \& Tudge, J. R. (2007) Responsividade materna em famílias de mães solteiras e famílias nucleares no terceiro mês de vida da criança. Estudos de Psicologia, 12(2), 109-117. Disponível em http://www.scielo.br/pdf/ epsic/v12n2/a02v12n2.pdf

Ribas A. F. P., \& Moura, S. M. L. (2004). Responsividade materna e teoria do apego: Uma discussão crítica do papel de estudos transculturais. Psicologia: Reflexão e Crítica, 17(3), 315-322.

Schmidt, E. B., Piccoloto, N. M., \& Müller, M. C. (2005). Depressão pós-parto: Fatores de risco e repercussões no desenvolvimento infantil. Psico USF, 10(1), 61-68.

Schwengber, D., \& Piccinini, C. (2003) O impacto da depressão pós-parto para interação mãe-bebê. Estudos de Psicologia, 8(3), 403-411.

Silva, F. C. S., Araújo, T. M., Araújo, M. F. M., Carvalho C. M. L., \& Caetano, J. A. (2010) Depressão pós-parto em puérperas: conhecendo interações entre mãe, filho e família. Acta Paulista de Enfermagem, 23 (3), 411-416.

Spitz, R. A. (1979). O primeiro ano de vida: Um estudo psicanalítico do desenvolvimento normal e anômalo das relações objetais. São Paulo: Martins Fontes.

Stern, D. (1997). A constelação da maternidade: O panorama da psicoterapia pais/bebê. Porto Alegre: Artes Médicas.

Tedesco, J. J. A., Quayle, J., \& Zugaib, M. (1997). Obstetrícia psicossomática. São Paulo: Atheneu.

Tostes, N. (2012). Percepção de gestantes acerca da assistência pré-natal, seus sentimentos e expectativas quanto ao preparo para o parto. Dissertação (Mestrado em Psicologia da Saúde, Universidade de Brasília, Brasília, DF). Disponível em http://repositorio.unb.br/ bitstream/10482/11099/1/2012_NataliaAlmeidaTostes.pdf

Winnicott, D. D. (1999). Privação e delinqüência. São Paulo: Martins Fontes. 
Recebido: $12 / 05 / 2014$

última revisão: 22/03/2017

Aceite final: 29/03/2017

\section{Sobre os autores:}

Adriane Diehl Krob: Doutoranda pela Universidad de Ciencias Empresariales y Sociales (UCES, 2014). Possui Graduação em Psicologia pela Pontifícia Universidade Católica do Rio Grande do Sul (1996), Mestrado em Psicologia do Desenvolvimento Humano pela Universidade Federal do Rio Grande do Sul (1999) e Especialização em Psicologia Hospitalar (Psicologia da Saúde) pelo Instituto de Educação e Pesquisa do Hospital Moinhos de Vento (2011). Tem experiência na área de Psicologia do Desenvolvimento, Psicologia Hospitalar e da Saúde, Psicologia Comunitária e Psicologia da Educação. Pesquisa e atua principalmente nos seguintes temas: gestação, amamentação, maternidade, paternidade, infância, adolescência e interação pais/bebê-filhos. E-mail: krobpsi@terra.com.br

Josehelen de Godoy: Graduada em Psicologia pela Faculdade Anglo Americano (FAA) em 2013. Atua como Psicóloga no Ambulatório e no Núcleo de Apoio à Saúde da Família. E-mail: josi_godoy_@hotmail.com

Keila Pamela Leite: Graduada em Psicologia pela Faculdade Anglo Americano (FAA) em 2013 e atua como Psicóloga Social no Centro de Referência de Assistência Social (CRAS). E-mail: keila_kpl@hotmail.com

Samantha Gottardo Mori: Graduada em Psicologia pela Faculdade Anglo Americano (FAA) em 2013, Pós-graduanda em Terapia Cognitiva Comportamental, pela União Dinâmica de Faculdades Cataratas (UDC) e atua como Psicóloga Clínica e Jurídica. E-mail: samanthagmori@gmail.com 